\title{
Corneal Transparency After Cross-linking for Keratoconus: 1-Year Follow-up
}

\author{
Ramon Gutiérrez, MD, PhD; Inma Lopez, MD; Cesar Villa-Collar, OD, PhD; \\ José Manuel González-Méijome, OD, PhD
}

\section{ABSTRACT}

PURPOSE: To evaluate the changes in corneal transparency and thickness measured objectively using Pentacam Scheimpflug (Oculus Optikgeräte $\mathrm{GmbH}$ ) corneal tomography (densitometry) after corneal cross-linking (CXL).

METHODS: Twenty-two eyes from 15 patients with keratoconus underwent CXL. Corneal thickness and curvature as well as lens and corneal densitometry were measured at baseline and 1, 3, 6, and 12 months after CXL with Pentacam.

RESULTS: Immediately after CXL, central corneal thickness significantly decreased $(-40 \mu \mathrm{m})$ and corneal densitometry increased significantly. At 6-month followup, densitometry values were reduced and seemed to stabilize at a higher value than baseline. No statistically significant differences were noted (mean difference at 12 months: $+30 \%)$. Changes in corneal densitometry were detected with the Pentacam even when clinical haze did not increase beyond grade 1 during follow-up.

CONCLUSIONS: Corneal CXL is associated with a transient reduction in central corneal thickness, which recovered to baseline after 12 months. During the same time period, corneal densitometry experienced a transient increase that recovered to baseline values after 3 months. Changes in compactness of the corneal tissue after CXL may have an impact in corneal power not due to curvature changes, but to refractive index changes as a result of hydration changes. Pentacam is a useful objective method to monitor corneal transparency after CXL. [J Refract Surg. 2012;28(11):781-785.] doi:10.3928/1081597X-20121011-06

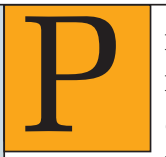

resently, corneal collagen cross-linking (CXL) with riboflavin $\mathrm{A}$ is the only pharmacological treatment available for keratoconus. ${ }^{1}$ This treatment is intended to reinforce the corneal architecture by cross-linking the stromal lamellae in a transversal way. ${ }^{2}$ Several studies have proven that the treatment is safe, effective, and provides the desired stability of the condition ${ }^{3}$ as well as improves several topographic indices and visual acuity compared with untreated eyes. ${ }^{4}$ Despite this, the mechanism by which the anterior corneal surface changes towards a flatter shape is not clear. Mechanical stretching of the corneal tissue could be related with the compaction of the collagen lamellae, thus thinning the cornea. ${ }^{5}$ However, it could also be argued that the entire cornea would stretch, thus exerting some backward movement that could be observed as a change in the posterior corneal surface and potentially on anterior chamber depth as measured with current Scheimpflug optical tomography. ${ }^{6}$

Transient corneal haze is a relatively common side effect of CXL and reflects the healing process of the cornea. ${ }^{7-10}$ Currently, haze can be measured objectively by optical methods such as Scheimpflug photography. ${ }^{11}$

The goal of this study was to objectively evaluate changes in central corneal thickness (CCT) and transparency after CXL in patients with keratoconus, as measured with Pentacam (Oculus Optikgeräte GmbH, Wetzlar, Germany).

From the Department of Surgery (Ophthalmology), University of Murcia, Murcia, Spain (Gutiérrez, Lopez); Clínica Oftalmológica Novovisión, Madrid, Spain (Gutiérrez, Villa-Collar); and Clinical \& Experimental Optometry Research Lab, Center of Physics (Optometry), School of Sciences, University of Minho, Braga, Portugal (González-Méijome).

This study was supported in part by a grant from the Spanish Ministry of Health, Instituto Carlos III, Red Temática de Investigación Cooperativa en Salud "Patología ocular del envejecimiento, calidad visual y calidad de vida," Subproyecto de Calidad Visual (RD07/0062)

The authors have no proprietary or financial interest in the materials presented herein.

Correspondence: José Manuel González-Méijome, OD, PhD, Clinical \& Experimental Optometry Research Lab, Center of Physics (Optometry), University of Minho, 4710-057 Braga, Portugal. Tel: 35125360 4320; Fax: 35125367 8981; E-mail: jgmeijome@fisica.uminho.pt

Received: January 13, 2012; Accepted: August 22, 2012 


\section{Corneal Transparency After CXL for Keratoconus/Gutiérrez et al}

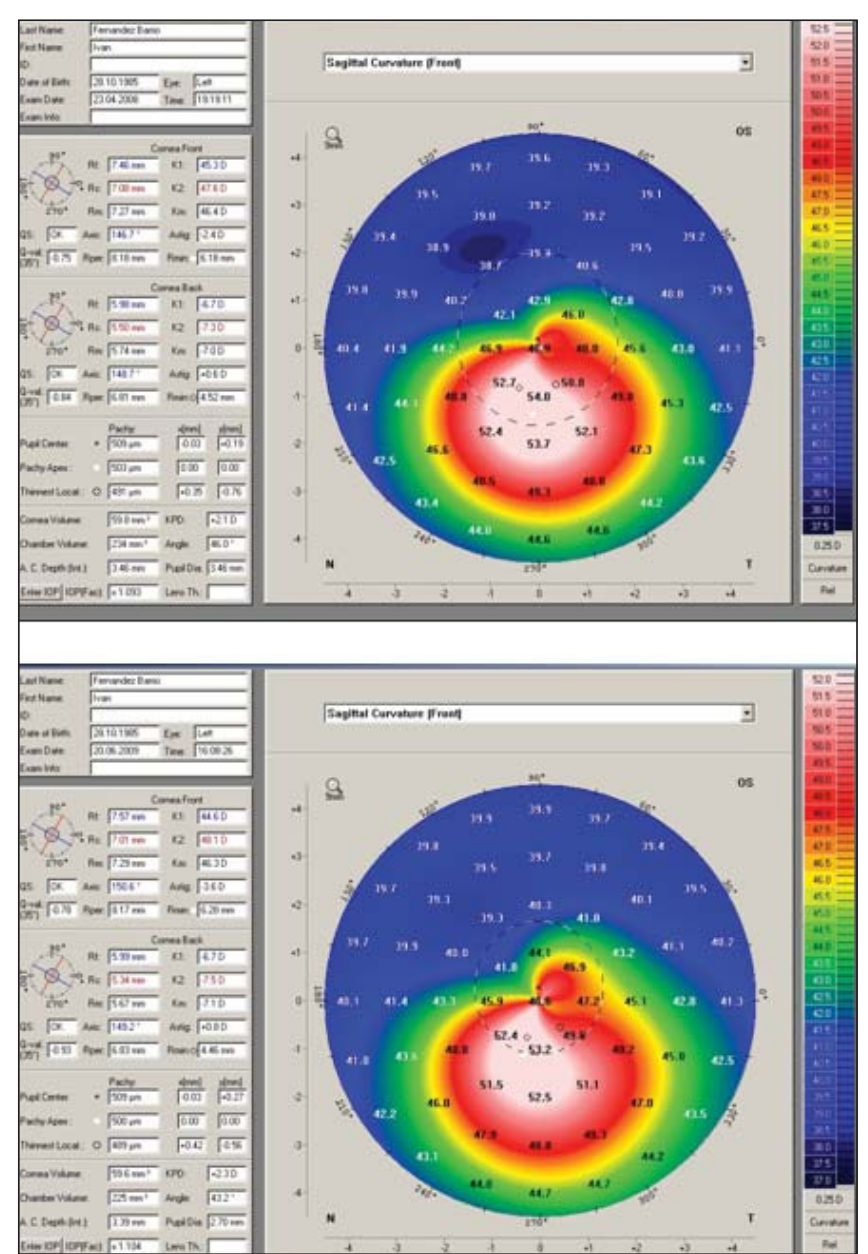

Figure 1. Topographic images before (top) and 2 months after cross-linking treatment (bottom).

\section{PATIENTS AND METHODS}

Twenty-two eyes from 15 patients with keratoconus were evaluated with Pentacam. To minimize the effect of diurnal changes in corneal hydration, all measurements were performed between 2:00 and 6:00 PM. ${ }^{12}$

Two days before treatment, patient eyelids were washed every 12 hours and a combination of dexamethasone and chloramphenicol was instilled three times a day.

Topical anesthesia of the cornea was obtained using oxybuprocaine and tetracaine drops, alternating every 3 minutes for 9 minutes. During this time period, pilocarpine $2 \%$ eye drops were also instilled twice. After a lid speculum was inserted, a 9.0-mm diameter corneal abrasion was created. Riboflavin $0.1 \%$ drops were instilled every 3 minutes for 30 minutes. The riboflavin drops were prepared immediately before treatment by mixing aqueous riboflavin $0.5 \%$ solution with dextran T-500 20\% solution. Central corneal pachymetry was performed using ultrasound. In eyes with a CCT (with- out epithelium) $<400 \mu \mathrm{m}$, additional riboflavin $0.1 \%$ drops without dextran (hypoosmotic drops) were applied until the thickness was $>400 \mu \mathrm{m}$. The eyes were inspected at the slit-lamp to ensure that the riboflavin had penetrated the aqueous (blue light).

The eye was irradiated for 30 minutes with an ultraviolet-A irradiance of $3 \mathrm{~mW} / \mathrm{cm}^{2}$ (UV-X; Peschke Meditrade $\mathrm{GmbH}$, Hunenberg, Switzerland). During irradiation, the cornea was moistened every 5 minutes with riboflavin $0.1 \%$ drops and oxybuprocaine drops every 10 minutes. Ofloxacin $0.3 \%$ ointment was applied at the end of the procedure and the eye was patched. Patients were informed to use the tobramycin drops every 2 hours for 5 days. After epithelial healing, patients used topical fluorometholone two times a day for 3 months. For eyes with sterile infiltrates during the early postoperative period, dexamethasone drops were prescribed five times a day for 1 week, after which the drops were tapered during the following 2 weeks. All procedures were uneventful.

Statistical analysis was conducted with SPSS 18.0 (SPSS Inc, Chicago, Illinois). Significance of changes over time was assessed using analysis of variance after assessing normality in the data distribution with the Shapiro-Wilks W-test. Changes at subsequent followup compared with baseline were assessed using paired sample $t$ tests. $P<.05$ was considered statistically significant.

\section{RESULTS}

Figure 1 is representative topographies before and 2 months after CXL. Figure 2 shows changes in CCT over 1 year of follow-up after CXL. A statistically significant decrease was noted immediately after treatment $(P<.01$ at 1 month and $P<.05$ at 3 and 6 months), returning to baseline values after 12 months $(P>.05)$. Figure 3 illustrates transient changes in corneal transparency showing a significant increase in corneal densitometry after 1 month and remaining stable up to 3 months $(P<.05$ against baseline) with reduction and stabilization of corneal transparency at 6 and 12 months postoperatively. These changes were present despite the absence of significant haze observed on slit-lamp examination (no patient showed values over grade 1 on clinical observation).

Table A (available as supplemental material in the PDF version of this article) presents the changes in clinical and morphological corneal parameters showing mild changes in keratoconus indices over the course of follow-up. Index of vertical asymmetry (Fig 4) and index of height asymmetry (Fig 5) at 1 and 3 months $(P<.05$ against baseline) returned to baseline values after 6 months. 


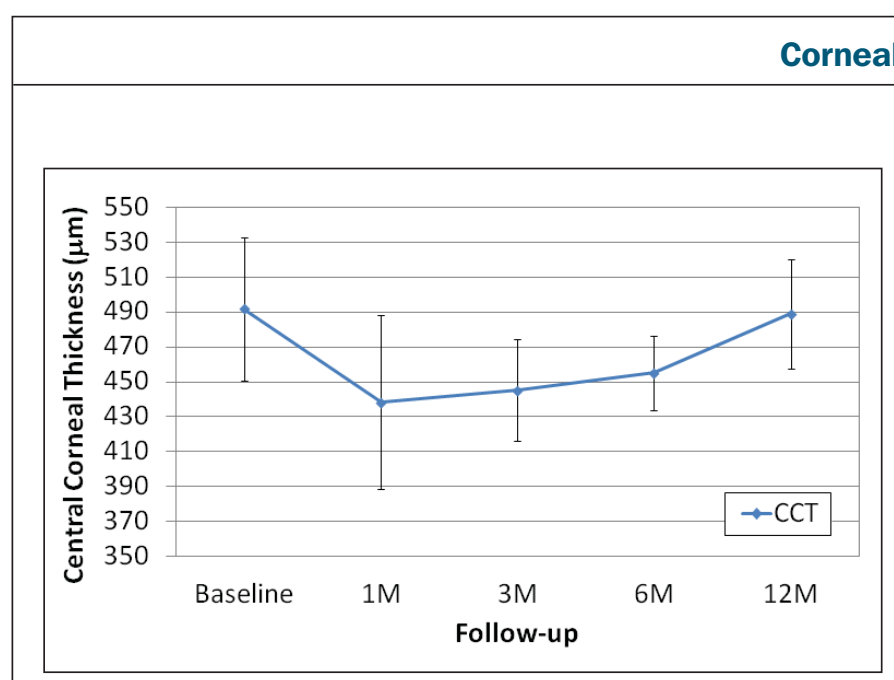

Figure 2. Change in central corneal thickness (CCT) measured at the geometric center with Pentacam.

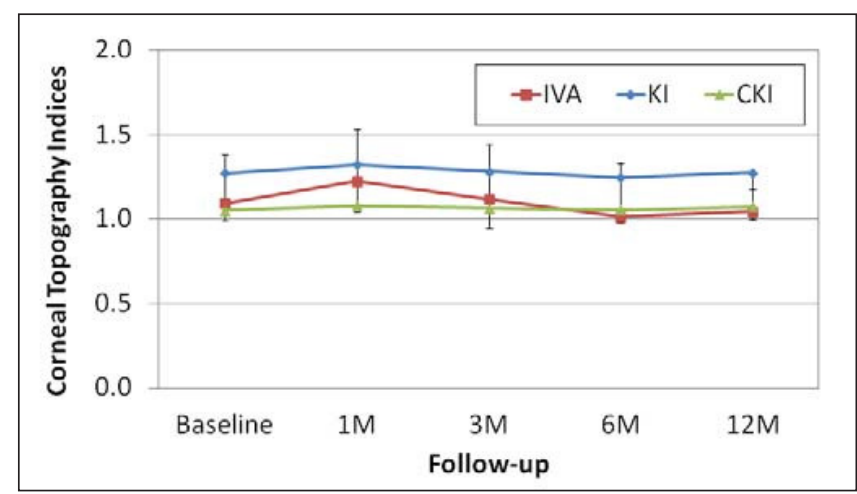

Figure 4. Changes in Pentacam index of vertical asymmetry (IVA), keratoconus index (KI), and center keratoconus index (CKI).

\section{DISCUSSION}

Transient haze is a common side effect of corneal CXL. This reaction is related to the healing process after treatment and is commonly graded subjectively by slitlamp examination, which may not detect mild changes between follow-up visits. This is considered a transient consequence of the histological changes induced by the alteration of the cross-linked structure of the stroma, but definitive changes have also been documented. ${ }^{8}$ Haze formation is a concern after CXL, as it can potentially limit the benefits of the treatment by diminishing the transparency of the cornea, thus limiting the potential visual acuity achievable by means of subsequent treatments ${ }^{13-15}$ or contact lens fitting. ${ }^{16}$

This study demonstrates that Pentacam densitometry measures are able to detect statistically significant changes in corneal transparency 1 year after CXL in patients with keratoconus. The significant increase and subsequent decrease in corneal densitometry after CXL in patients with keratoconus agrees with a study from Greenstein et $\mathrm{al}^{11}$ reporting the reduction of haze graded clinically with the slit-lamp from grade 0.78 to

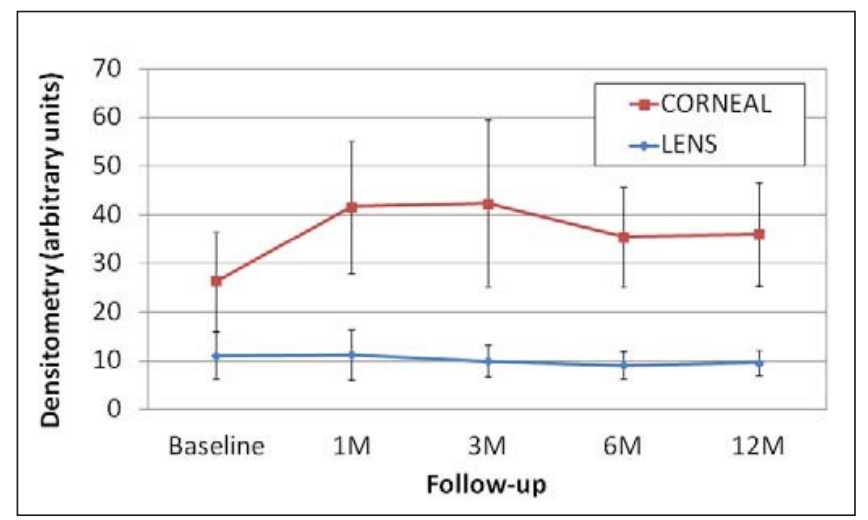

Figure 3. Change in corneal and lens densitometry measured with Pentacam. $P<.001$ at 1 and 3 months compared to baseline for corneal densitometry and $P=.014$ between 1 and 3 months for lens densitometry.

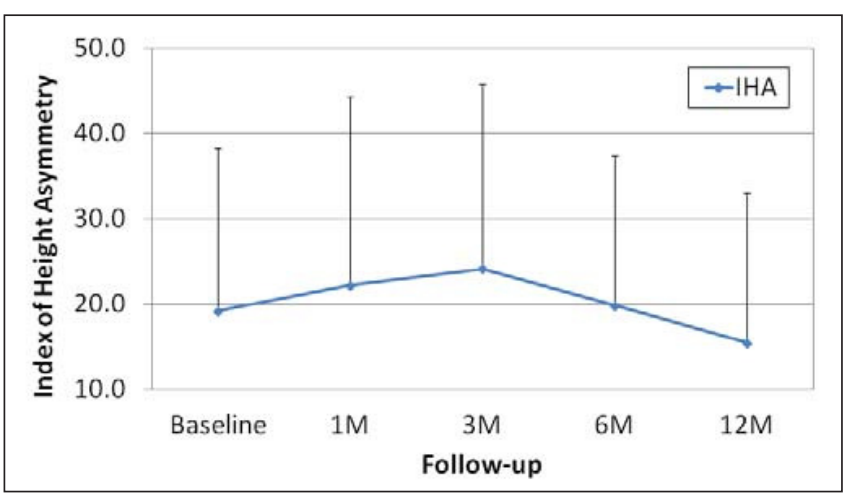

Figure 5. Changes in Pentacam index of height asymmetry (IHA).

0.061 year after treatment. However, values observed in the present study are markedly higher despite patients not showing clinical haze over grade 1 on slitlamp examination.

It is well known that haze related with CXL is different from haze related with other treatments such as photorefractive keratectomy (PRK). Although PRKinduced haze is limited to the subepithelial area, CXLrelated haze achieves up to $60 \%$ of the stromal depth. There have been several speculations regarding the etiology of haze in CXL and several studies have related it to loss of keratocytes. ${ }^{9}$ Other potential explanations may relate this occurrence with the histological changes in terms of collagen structure. After CXL, the new molecular bridges established between collagen lamellae may affect the organization of the stromal structure responsible for corneal transparency. This effect is seen by evaluating the changes in corneal thickness immediately after treatment where marked thinning is present. Although haze and thinning are both related with the healing process and collagen reorganization into the corneal stroma, the time period 
Corneal Transparency After CXL for Keratoconus/Gutiérrez et al

of change is markedly different, with corneal transparency recovering faster than corneal thickness.

The transient increase in some keratoconus indices is potentially related with the structural changes taking place within the cornea, which is reflected in the present study by a transient statistically significant increase in keratoconus grading and an increase in the index of height asymmetry; although this was not statistically significant due to the higher variability in this parameter. The structural changes taking place after CXL may have significant implications in terms of refraction mediated by the potential changes in the refractive index of the cornea.

Unless a loss of collagen lamellae could be demonstrated, which is not likely as this will potentially result in weak corneal tissue after CXL for keratoconus, changes in corneal thickness seem to reflect a compactness of corneal stroma. We assume that keratocyte ${ }^{9}$ and fundamental substance loss will result in a significant loss of hydration and consequently a decrease in refractive index. A recent study has shown that CXL is related to increased keratocyte apoptosis, followed by a process of regeneration that may explain the transient haze noted after CXL. ${ }^{17}$ Another explanation of the thickness changes could be a misinterpretation of the anterior and posterior edges of the cornea by Pentacam as a result of the changes in induced transparency. However, in our patients, we ensured that all images were processed correctly and no misdetection of the posterior border occurred, thus we ruled out this potential source of error for this sample. Moreover, other nonoptical techniques have obtained similar or higher thinning results. Holopainen and Krootila, ${ }^{5}$ using ultrasound pachymetry, observed an average thinning of $87 \mu \mathrm{m}$ just after treatment, which recovered to baseline after 6 months. It seems that the reduction in corneal thickness measured with ultrasound pachymetry following CXL may reflect a true thinning effect of the treatment, thus supporting the Pentacam results.

Corneal thickness changes may be relevant in the understanding of the refractive changes in CXL. Assuming an increase in the compactness of the dry tissue, this will eventually result in an increase in the refractive index of the cornea. Leonard and Meek ${ }^{18}$ obtained refractive indices of 1.411 and 1.365 for the fibrils and extrafibrillar material, respectively, in human corneal stroma and found that areas of more compactness, such as the center and anterior stroma, ${ }^{19}$ have lower refractive index, likely due to a higher proportion of extrafibrillar material. ${ }^{20}$ This effect will be combined with the presence of a thinner corneal tissue. However, a decrease in corneal thickness of $100 \mu \mathrm{m}$ will have minor impact in the overall power of the cornea $(<0.01 \mathrm{D})$. Changes in corneal hydration as a consequence of the CXL procedure could have greater refractive implications. Let us consider the known relationship between hydration and refractive index of hydrophilic soft materials (HEMA-based) as an example to apply here. In this case, the refractive index of the cornea could change from 1.376 (assuming 78\% corneal hydration or soft hydrogel Vasurfilcon A with equilibrium water content of $74 \%$ and a refractive index of 1.379) to 1.415 (corresponding to Etafilcon A material with $55 \%$ equilibrium water content assuming that a $100-\mu \mathrm{m}$ reduction in CCT will result in approximately $20 \%$ to $25 \%$ volume loss). This increase in refractive index will also increase the overall power of the cornea in the myopic direction by approximately 6.00 to $7.00 \mathrm{D}$ for a $6.5-\mathrm{mm}$ corneal curvature in the anterior surface and 5.5-mm curvature in the posterior corneal surface (greater effect could be expected for steeper corneas). This effect needs to be verified, but may limit the ability of CXL treatment in the earlier stages. In the long-term, a normalization of the hydration of the cornea and the flattening effect induced by the corneal healing will result in better visual and refractive results or even in changes in the hyperopic direction when refractive index no longer plays a role and corneal flattening induces a reduction in the myopic power of the eye typical in patients with keratoconus. The same calculations for a reduction in CCT of $50 \mu \mathrm{m}$ are in agreement with our results (reduction of approximately $10 \%$ to $15 \%$ in CCT resulting in a shift in refractive index from 1.376 to 1.39 typical of Alphafilcon A material with $66 \%$ equilibrium water content), impacting the overall power of the cornea from 2.00 to $3.00 \mathrm{D}$ in the myopic direction, which also agrees with results commonly found after CXL. The refractive indices of hydrogel materials were considered from previously published results. ${ }^{21,22}$ Furthermore, changes in the refractive index of the cornea may induce errors in keratometric and keratoscopic devices, but this is less likely considering that the detection of the anterior corneal surface (tear film and epithelium) will not be affected by stromal hydration changes and healing processes subsequent to CXL treatment.

One limitation of the current study is that we do not know the actual value of the corneal thickness because only measurements with Pentacam were obtained. Due to the increased densitometry, the image quality of the optical sections obtained with the Pentacam may create artifacts affecting the ability of the image analysis system to detect the edges of the cornea, thus reporting abnormal thickness values. However, the time period of changes in thickness and keratoconus does not justify this behavior because even after the cornea has 
recovered transparency, CCT did not recover to normal values. This is consistent with a previous report on transient thinning of the cornea with ultrasound pachymetry after CXL treatment. ${ }^{5}$

The slight reduction in lens densitometry seems to be related to the increasing absorption and diffusion of light by the cornea. In fact, there is not a cause for the transient increase in lens densitometry so this finding seems to be the result of an artifact due to increasing light absorption by the corneal tissue after CXL. Nevertheless, changes in lens densitometry were not statistically significant during follow-up.

The present study demonstrates the impact of CXL in two fundamental properties of the corneal histology-CCT, which reflects the compactness of the cornea after treatment, recovering to baseline values after 12 months, and transparency, which may reflect changes in the histological distribution of stromal lamellae that recovers 3 months after treatment. These changes in corneal compactness may be associated with the myopic transient effect found in patients after CXL. The automatic measure using Pentacam is able to detect subtle degrees of opalescence even in mild cases of haze as graded clinically through slit-lamp examination. Further improvements in the densitometry function, possibly measuring the entire area of increased density and integrating all density measures across the corneal volume, will make this objective measure more representative of the relevant clinical features.

\section{AUTHOR CONTRIBUTIONS}

Study concept and design (R.G.); data collection (R.G., I.L., C.V.C., J.M.G.M.); analysis and interpretation of data (R.G., I.L., C.V.C., J.M.G.M.); drafting of the manuscript (R.G., J.M.G.M.); critical revision of the manuscript (R.G., I.L., C.V.C., J.M.G.M.); obtained funding (R.G.); supervision (R.G., I.L., C.V.C., J.M.G.M.)

\section{REFERENCES}

1. Wollensak G, Spoerl E, Seiler T. Riboflavin/ultraviolet-a-induced collagen crosslinking for the treatment of keratoconus. Am J Ophthalmol. 2003;135(5):620-627.

2. Wollensak G, Spörl E, Seiler T. Treatment of keratoconus by collagen cross linking. [German]. Ophthalmologe. 2003;100(1):44-49.

3. Koller T, Iseli HP, Hafezi F, Vinciguerra P, Seiler T. Scheimpflug imaging of corneas after collagen cross-linking. Cornea. 2009;28(5):510-515.

4. Coskunseven E, Jankov MR II, Hafezi F. Contralateral eye study of corneal collagen cross-linking with riboflavin and UVA irradiation in patients with keratoconus. J Refract Surg. 2009;25(4):371-376.

5. Holopainen JM, Krootila K. Transient corneal thinning in eyes undergoing corneal cross-linking. Am J Ophthalmol. 2011;152(4):533-536.
6. Edmonds CR, Wung SF, Pemberton B, Surrett S. Comparison of anterior chamber depth of normal and keratoconus eyes using Scheimpflug photography. Eye Contact Lens. 2009;35(3):120122.

7. Herrmann CI, Hammer T, Duncker GI. Haze formation (corneal scarring) after cross-linking therapy in keratoconus [German]. Ophthalmologe. 2008;105(5):485-487.

8. Raiskup F, Hoyer A, Spoerl E. Permanent corneal haze after riboflavin-UVA-induced cross-linking in keratoconus. J Refract Surg. 2009;25(9):S824-S828.

9. Mazzotta C, Balestrazzi A, Baiocchi S, Traversi C, Caporossi A. Stromal haze after combined riboflavin-UVA corneal collagen cross-linking in keratoconus: in vivo confocal microscopic evaluation. Clin Experiment Ophthalmol. 2007;35(6):580-582.

10. Kymionis GD, Portaliou DM, Diakonis VF, et al. Posterior linear stromal haze formation after simultaneous photorefractive keratectomy followed by corneal collagen cross linking. Invest Ophthalmol Vis Sci. 2010;51(10):5030-2033.

11. Greenstein SA, Fry KL, Bhatt J, Hersh PS. Natural history of corneal haze after collagen cross-linking for keratoconus and corneal ectasia: Scheimpflug and biomicroscopic analysis. J Cataract Refract Surg. 2010;36(12):2105-2114.

12. du Toit R, Vega JA, Fonn D, Simpson T. Diurnal variation of corneal sensitivity and thickness. Cornea. 2003;22(3):205-209.

13. Kanellopoulos AJ. Comparison of sequential vs same-day simultaneous collagen cross-linking and topography-guided PRK for treatment of keratoconus. J Refract Surg. 2009;25(9):S812-S818.

14. El-Raggal TM. Sequential versus concurrent Kerarings insertion and corneal collagen cross-linking for keratoconus. $\mathrm{Br} \mathrm{J} \mathrm{Oph-}$ thalmol. 2010;95(1):37-41.

15. Alfonso JF, Fernández-Vega L, Lisa C, Fernandes P, GonzálezMéijome JM, Montés-Micó R. Collagen copolymer toric posterior chamber phakic intraocular lens in eyes with keratoconus. J Cataract Refract Surg. 2010;36(6):906-916.

16. González-Méijome JM, Jorge J, de Almeida JB, Parafita MA. Soft contact lenses for keratoconus: case report. Eye Contact Lens. 2006;32(3):143-147.

17. Salomão MQ, Chaurasia SS, Sinha-Roy A, et al. Corneal wound healing after ultraviolet-A/riboflavin collagen cross-linking: a rabbit study. J Refract Surg. 2011;27(6):401-407.

18. Leonard DW, Meek KM. Refractive indices of the collagen fibrils and extrafibrillar material of the corneal stroma. Biophys J. 1997;72(3):1382-1387.

19. Winkler M, Chai D, Kriling S, et al. Nonlinear optical macroscopic assessment of 3-D corneal collagen organization and axial biomechanics. Invest Ophthalmol Vis Sci. 2011;52(12):88188827.

20. Vasudevan B, Simpson TL, Sivak JG. Regional variation in the refractive-index of the bovine and human cornea. Optom Vis Sci. 2008;85(10):977-981.

21. González-Méijome JM, Lira M, López-Alemany A, Almeida JB, Parafita MA, Refojo MF. Refractive index and equilibrium water content of conventional and silicone hydrogel contact lenses. Ophthalmic Physiol Opt. 2006;26(1):57-64.

22. González-Méijome JM, López-Alemany A, Lira M, Almeida JB, Oliveira ME, Parafita MA. Equivalences between refractive index and equilibrium water content of conventional and silicone hydrogel soft contact lenses from automated and manual refractometry. J Biomed Mater Res B Appl Biomater. 2007;80(1):184-191. 
TABLE A

Parameters Evaluated After CXL in Patients With Keratoconus

\begin{tabular}{|c|c|c|c|c|c|c|}
\hline Parameter & $\begin{array}{c}\text { Baseline } \\
(n=22)\end{array}$ & $\begin{array}{l}1 \text { Month } \\
(n=22)\end{array}$ & $\begin{array}{l}3 \text { Months } \\
(n=20)\end{array}$ & $\begin{array}{l}6 \text { Months } \\
(n=20)\end{array}$ & $\begin{array}{l}12 \text { Months } \\
(n=20)\end{array}$ & $P$ Value* \\
\hline Sphere (D) & $-2.96 \pm 2.41$ & $-2.78 \pm 0.26$ & $-2.67 \pm 3.25$ & $-2.58 \pm 3.33$ & $-2.63 \pm 2.63$ & $>.05$ \\
\hline Cylinder (D) & $2.95 \pm 2.30$ & $2.45 \pm 2.20$ & $3.42 \pm 2.35$ & $3.19 \pm 2.13$ & $3.22 \pm 1.71$ & $>.05$ \\
\hline CDVA (decimal) & $0.70 \pm 0.26$ & $0.77 \pm 0.29$ & $0.73 \pm 0.19$ & $0.78 \pm 0.26$ & $0.79 \pm 0.25$ & $<.05$ \\
\hline CCT $(\mu \mathrm{m})$ & $491 \pm 41$ & $438 \pm 50 \dagger$ & $445 \pm 29 \dagger$ & $455 \pm 22 \dagger$ & $489 \pm 31$ & $<.001$ \\
\hline $\begin{array}{l}\text { Maximum anterior } \\
\text { elevation }(\mu \mathrm{m})\end{array}$ & $36 \pm 17$ & $40 \pm 19$ & $35 \pm 16$ & $32 \pm 16$ & $42 \pm 23$ & $>.05$ \\
\hline $\begin{array}{l}\text { Maximum posterior } \\
\text { elevation }(\mu \mathrm{m})\end{array}$ & $64 \pm 29$ & $70 \pm 31$ & $66 \pm 24$ & $68 \pm 27$ & $79 \pm 37$ & $>.05$ \\
\hline $\mathrm{Q}\left(\right.$ at $30^{\circ}$ ) (anterior) & $-0.62 \pm 0.75$ & $-0.78 \pm 0.91$ & $-0.93 \pm 0.71$ & $-1.02 \pm 0.73$ & $-0.56 \pm 1.22$ & $>.05$ \\
\hline $\mathrm{Q}$ (at 30) (posterior) & $-0.69 \pm 0.60$ & $-0.92 \pm 0.64$ & $-0.80 \pm 0.63$ & $-0.77 \pm 0.66$ & $-0.89 \pm 0.63$ & $>.05$ \\
\hline $\begin{array}{l}\text { ACD at center } \\
(\mathrm{mm})\end{array}$ & $3.37 \pm 0.29$ & $3.44 \pm 0.31$ & $3.42 \pm 0.32$ & $3.40 \pm 0.32$ & $3.44 \pm 0.13$ & $>.05$ \\
\hline $\begin{array}{l}\text { ACD at maximum } \\
\text { posterior elevation } \\
(\mathrm{mm})\end{array}$ & $3.17 \pm 0.28$ & $3.17 \pm 0.28$ & $3.24 \pm 0.33$ & $3.26 \pm 0.27$ & $3.29 \pm 0.27$ & $>.05$ \\
\hline AC volume $\left(\mathrm{mm}^{3}\right)$ & $207 \pm 35$ & $216 \pm 35$ & $211 \pm 39$ & $206 \pm 40$ & $205 \pm 22$ & $>.05$ \\
\hline $\begin{array}{l}\text { Grade of } \\
\text { keratoconus }\end{array}$ & $2.57 \pm 0.88$ & $2.76 \pm 0.77 \dagger$ & $2.62 \pm 0.70$ & $2.50 \pm 0.71$ & $2.56 \pm 0.88$ & $<.05$ \\
\hline ISV & $99.05 \pm 43.25$ & $113.79 \pm 46.34$ & $102.89 \pm 35.90$ & $94.47 \pm 33.07$ & $105.11 \pm 59.09$ & $>.05$ \\
\hline IVA & $1.09 \pm 0.59$ & $1.22 \pm 0.61$ & $1.12 \pm 0.53$ & $1.01 \pm 0.48$ & $1.05 \pm 0.85$ & $>.05$ \\
\hline $\mathrm{KI}$ & $1.27 \pm 0.17$ & $1.32 \pm 0.18$ & $1.28 \pm 0.15$ & $1.25 \pm 0.15$ & $1.28 \pm 0.22$ & $>.05$ \\
\hline CKI & $1.05 \pm 0.05$ & $1.08 \pm 0.06$ & $1.07 \pm 0.06$ & $1.06 \pm 0.06$ & $1.07 \pm 0.05$ & $>.05$ \\
\hline $\mathrm{IHA}$ & $19.23 \pm 19.12$ & $22.21 \pm 22.13$ & $24.17 \pm 21.60$ & $19.83 \pm 17.59$ & $15.47 \pm 17.55$ & $>.05$ \\
\hline IHD & $0.08 \pm 0.06$ & $0.10 \pm 0.07$ & $0.09 \pm 0.06$ & $0.07 \pm 0.06$ & $0.07 \pm 0.06$ & $>.05$ \\
\hline $\mathrm{Rmin}(\mathrm{mm})$ & $5.95 \pm 0.72$ & $5.73 \pm 0.74$ & $5.83 \pm 0.71$ & $5.98 \pm 0.72$ & $5.83 \pm 0.77$ & $>.05$ \\
\hline \multicolumn{7}{|c|}{$\begin{array}{l}\text { CDVA = corrected distance visual acuity, CCT = central corneal thickness, } Q=\text { asphericity, } A C D=\text { anterior chamber depth, AC = anterior chamber, ISV }=\text { index } \\
\text { of surface variance, IVA = index of vertical asymmetry, } K I=\text { keratoconus index, CKI = center keratoconus index, IHA = index of height asymmetry, IHD = index o } \\
\text { height decentration, Rmin = minimum sagittal curvature } \\
\text { *Analysis of variance. } \\
\text { †Statistically significant compared to baseline. } \\
\text { Keratoconus grading was performed automatically according to the Pentacam software. }\end{array}$} \\
\hline
\end{tabular}

